\title{
Meckern auf hohem Niveau?
}

Die medizinische Versorgung sei in Gefahr, klagen viele, und alles werde immer teurer. Doch Schweizer Patienten geht es im Vergleich zu anderen Ländern sehr gut. Aber der finanzielle Druck steigt. Was getan werden muss, um bei knapperen Ressourcen die medizinische Versorgung weiterhin auf hohem Niveau halten zu können, diskutierten kürzlich Experten aus Medizin, Industrie und Politik im Rahmen des 10. CARTA-Cardiovascular Roundtable Meetings in Zürich, organisiert vom Zurich Heart House. Das Fazit: Die Schweiz verfügt über viele Ressourcen, die aber nicht optimal genutzt werden.

Felicitas Witte

Dr. med., Medizinjournalistin

Korrespondenz: Dr. med. Felicitas Witte Pfeffingerstrasse 37 CH-4053 Basel

felicitas.witte[at]web.de
Kranke Menschen in der Schweiz haben Angst: Immer mehr sollen sie zu Medikamenten dazubezahlen, Ärzte und Pflegepersonal haben keine Zeit mehr, weil Stellen gestrichen werden, und neue Therapien bekommen nur Privatversicherte. Stimmt das wirklich? «Es gibt immer wieder Leute, die unser Gesundheitssystem schlechtmachen», sagte Bernhard Wegmüller, Direktor bei $\mathrm{H}+$ und Mitglied im Verwaltungsrat der SwissDRG AG, «aber so schlecht geht es uns nicht.» 10,7 Prozent des Bruttosozialproduktes gibt die Schweiz pro Jahr für die Gesundheit aus. Mehr investieren nur die USA und Frankreich, der Durchschnitt in den OECD-Ländern liegt bei 9,0 Prozent. Doch der Druck steigt. Die DRG-Fallpauschalen im stationären Bereich erhöhen auch hierzulande den Kostendruck, und im ambulanten Bereich hat der «Verteilungskampf» zwischen Hausärzten auf der einen und Spezialisten und Spitälern auf der anderen Seite begonnen. Die Kantone müssen mit immer knapperen Mitteln auskommen, dabei verursachen neue Therapien und die immer älter werdende Bevölkerung steigende Kosten. Die Schweizer gehen vernünftig mit ihren Ressourcen um, so Wegmüller, aber man würde sie nicht optimal nutzen. So könnte vielfach die Infrastruktur verbessert werden, Prozesse standardisiert oder Aufgaben neu verteilt werden. In anderen Ländern gäbe es das schon seit längerem. In den USA wurden zum Beispiel schon vor Jahren die Advanced Practice Nurses eingeführt, Pflegende mit einer speziellen Zusatzausbildung, etwa für Wund-Management oder Hypertonie-Behandlung. In ihrem Fachgebiet wissen sie oft mehr als der Arzt und können ihn entlasten. In Deutschland gibt es Chirurgisch Technische Assistenten, die «schneiden und nähen», während der Chirurg nur für die Operation kommt.

\section{Rahmenbedingungen für den geänderten Arztberuf schaffen}

Schweizer Ärzte klagen über lange Arbeitszeiten und viel Stress, aber im Vergleich zu anderen Ländern

\section{Cardiovascular Roundtable}

Nombreux sont ceux qui craignent que les soins médicaux soient menacés et se plaignent de prix toujours plus élevés. Mais la situation des patients suisses est excellente par rapport à ce qui se passe dans d'autres pays. Toutefois, la pression financière s'accroît. Récemment, dans le cadre de la dixième Cardiovascular Roundtable à Zurich organisée par le Zurich Heart House, des experts des milieux médical, industriel et politique ont évoqué les mesures nécessaires pour maintenir le niveau élevé des soins médicaux en dépit de la diminution des ressources. Leur conclusion: la Suisse dispose de nombreuses ressources dont l'utilisation n'est cependant pas optimale.

geht es ihnen gut. 3,8 Ärzte kümmern sich hierzulande um 1000 Einwohner, in den USA sind es nur 2,4, und im OECD-Durchschnitt 3,1. Das Berufsbild des Arztes habe sich aber geändert, und darauf müsse man eingehen. Mehr Teilzeitangebote, WorkLife-Balance verbessern, Wiedereinstiegsmöglichkeiten für Frauen nach der Babypause ermöglichen und dafür sorgen, dass auch Chefs geregelte Arbeitszeiten oder Teilzeitarbeit gut finden. Statt aus dem Ausland Ärzte zu importieren, sollte die Schweiz mehr eigene Ärzte ausbilden. «Dann muss aber auch die Politik etwas tun und bessere Rahmenbedingungen dafür schaffen», sagte Thomas F. Lüscher, Direktor der Kardiologie am Unispital Zürich. Er habe zurzeit vier schwangere Mitarbeiterinnen ohne Unterstützung für den Arbeitskraftausfall. Auch hätte er ohne «Import» von Ärzten aus dem Ausland nicht alle Stellen besetzen können. Das Aus- und Weiterbildungssystem der Ärzte müsse man kritisch hinterfragen, forderte Wegmüller. «Kann man von Medizinstudenten nicht erwarten, dass sie sich bei einer so teuren Ausbildung schon früher für eine Spezialisierung entscheiden?», fragte Wegmüller provokant. So könnte es eine kürzere «Grundausbildung» für alle geben, gefolgt von der Spezialausbildung für die ver- 


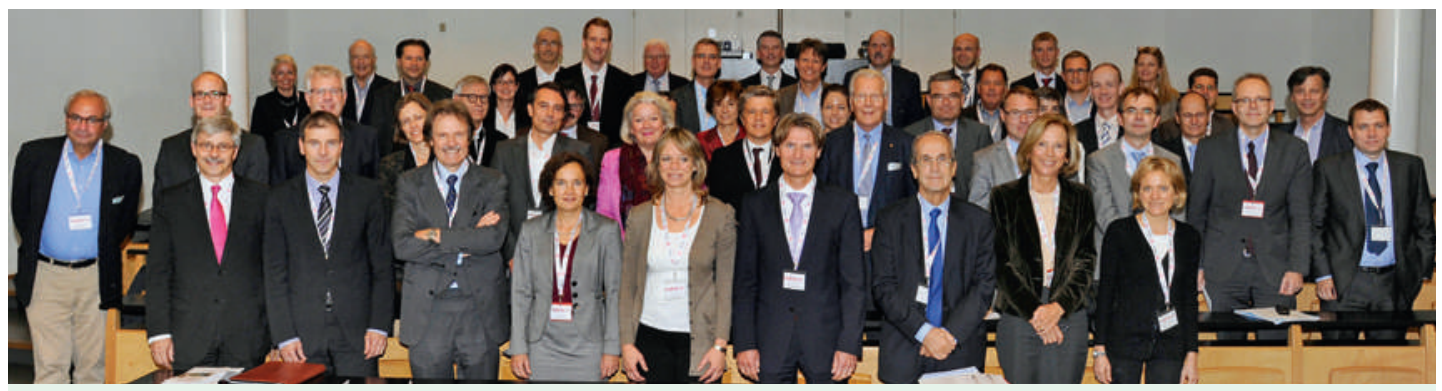

Die Teilnehmer am 10. Cardiovascular Roundtable im Zürcher UniversitätsSpital.

schiedenen Fachärzte. Er fordere das schon seit Jahren, sagte Johann Steurer, Leiter des Horten Zentrums für praxisorientierte Forschung und Wissenstransfer am Unispital Zürich. «Braucht ein Augenarzt in einer Praxis, der nur Katarakte anschaut und Fehlsichtigkeit behandelt, wirklich eine Ausbildung von zehn Jahren? Es ist doch Ressourcen-Verschwendung, wenn man Leute ausbildet, und diese Sachen lernen müssen, die sie später nie wieder brauchen.» Es gäbe nicht mehr eine Medizin, sondern mindestens 50 - das müsse sich auch im Studium abbilden. Dass einige so lange brauchen, liegt auch an der hohen Spitaldichte der Schweiz. «Am meisten lernt man, wenn man im Spital gebraucht wird und dort viele Eingriffe durchgeführt werden», sagte Lüscher. «Durch die neuen kürzeren Arbeitszeiten in der Weiterbildung ist es zusätzlich schwieriger, genügend Eingriffe zu machen.»

An das «Wesen der Schweiz» müsse man sich wieder erinnern, um die Ressourcen im Schweizer Gesundheitssystem zu sichern, wenn es nach Ignazio Cassis geht. Der Mediziner ist Fraktions-Vizepräsident der FDP und sitzt für den Kanton Tessin im Nationalrat. Anders als in Frankreich, Italien oder in Grossbritannien gäbe es in der Schweiz 27 Gesundheitssysteme. «Das Dilemma ist, dass $26 \mathrm{Mal}$ in den Kantonen bestimmt wird, welche Struktur die Systeme haben sollen, aber gezahlt wird aufgrund eines zwei wichtige Strukturänderungen geschaffen, zum einen SwissDRG, zum anderen Managed Care, das jetzt als integrierte Versorgung weitergeführt werde. «Wir müssen uns überlegen, wie viel Regulierung wir wollen.» Solle etwa die Politik entscheiden, wie welcher Patient betreut wird, etwa mit staatlichen Disease Management-Programmen wie in Deutschland, oder solle sie nur richtige Rahmenbedingungen schaffen, wie finanzielle Anreize? Würden sich zum Beispiel immer mehr Ärzte vernetzen, so Cassis, könnten sie einen enormen finanziellen Druck auf die Versicherer ausüben.

\section{Mehr und bessere ambulante Strukturen für die Patienten der Zukunft}

Immer mehr gibt die Schweiz für die Gesundheit aus: 1970 waren es nur sechs Prozent des Bruttoinlandproduktes, heute ist es doppelt so viel. Man dürfe sich von den Zahlen aber nicht täuschen lassen, erklärte Stefan Felder, Professor für Gesundheitsökonomie an der Uni Basel. «Im Vergleich zur übrigen Wirtschaft ist der Gesundheitsbereich wenig produktiv.» So hängt die Produktivitätsentwicklung im Gesundheitssektor OECD-weit pro Jahr drei Prozent hinter der allgemeinen Entwicklung zurück. «Der Gesundheitssektor ist nicht so ein starker Motor der Wirtschaft, wie viele immer denken», sagte Felder. Dabei gebe es deutliche Unterschiede zwi-

\section{«Der Gesundheitssektor ist nicht so ein starker Motor der Wirtschaft,} wie viele immer denken.» Stefan Felder

Articles

interactifs

Vous souhaitez commenter cet article? II vous suffit pour cela d'utiliser la fonction «Ajouter un commentaire» dans la version en ligne. Vous pouvez également consulter les remarques de vos confrères sous: www.bullmed.ch/ numero-actuel/ articles-interactifs/
Versicherungsgesetzes, das der Bund bestimmt.» Das liberale Gesundheitssystem sei die Lösung, in dem nicht der Staat agiert, sondern die Leistungserbringer und die Versicherer. In den letzten Jahren waren diese beiden aber immer weniger in der Lage, Lösungen zu finden, umso mehr hätte der Staat eingegriffen. Auf der Produktebene, das heisst mit Medikamenten oder Medizinprodukten, sei die Schweiz gut dabei. «Auf der Systemebene funktionieren wir aber nicht viel anders als vor hundert Jahren», sagte Cassis. «Viele wollen ja nichts ändern - sie haben offensichtlich Angst, dass in einem optimierten System weniger Geld fliesst.» Das Parlament habe trotzdem schen den einzelnen Bereichen im Gesundheitssektor: Die Pharmaindustrie ist sehr produktiv, Krankenhäuser, der ambulante Bereich, Heime und Sozialwesen tragen dagegen nur wenig zur Produktivität bei. Ohne den Pharmabereich wäre der Wachstumsbeitrag des Gesundheitssektors seit 1997 negativ. Überraschend waren Erklärungen des Professors, dass die demographische Entwicklung keine so grosse Rolle für die steigenden Gesundheitsausgaben spielt wie oft vermutet wird. «Die Gesundheitsversorgung älterer Menschen kostet zwar mehr, aber der Einfluss wird bei weitem überschätzt», sagte Felder. Viel wichtiger sei die Einkommensentwicklung: 
Werden Menschen reicher, geben sie mehr Geld für Gesundheit aus. Der medizinisch-technische Fortschritt erhöhe zwar auch die Kosten, die neuen Therapien würden aber nicht umgesetzt, wenn die Nachfrage nicht gestiegen wäre.

Das Patientenbild der Zukunft wird sich ändern, und darauf müsse man sich einstellen, sagte Carlo Conti, Vorsteher des Gesundheitsdepartements in Basel-Stadt und Präsident der Gesundheitsdirektorenkonferenz. Es werde mehr ältere, multimorbide Patienten geben, mehr Menschen mit Demenz, die stationäre Langzeitpflege brauchen. Immer mehr Gesundheitsleistungen könnten zudem ambulant erfolgen. Darauf müsse die Politik reagieren und mehr ambulante und integrierte Versorgungsmodelle fördern.» Das dürfe jedoch nicht zulasten der Versicherten gehen. «Es findet eine Verschiebung vom dual-finanzierten stationären in den von den Versicherten alleine finanzierten ambulanten Bereich statt», sagte Conti. «Die Prämien werden explodieren und der Einzelne muss immer mehr zahlen.»

\section{Mit schwerkranken Patienten über das Lebensende sprechen}

$\mathrm{Zu}$ wenig diskutieren Ärzte und die Öffentlichkeit den Umgang mit schwerkranken Patienten vor dem Lebensende. 80 Prozent der gesamten stationären Kosten in der Schweiz fallen im letzten Lebensjahr an, rechnete Conti vor. «Wir geben unendlich viel dafür aus, um das Leben um wenige Monate zu verlängern. Soll am Lebensende wirklich alles gemacht werden, was technisch möglich ist?» Thomas Lüscher steht im Alltag häufig vor dieser schwierigen Entscheidung. Er berichtete von einer 66-jährigen Patientin, die kürzlich mit Verdacht auf Herzinfarkt eingeliefert wurde. Der Kardiologe wollte eine PTCA

\section{«Frage ich meine Assistenten, will kaum einer eine kardiologische Praxis. Die meisten wollen Leitende oder Chefärzte werden.» Thomas F. Lüscher}

machen, es stellte sich aber heraus, dass sie eine Aortendissektion hatte und der gesamte Aorta-Hauptstamm verschlossen war. «Die Prognose war extrem schlecht, aber die Frau hat noch klar mit uns gesprochen», erzählte Lüscher. «Solche Situationen erleben wir regelmässig und wir müssen dann entscheiden das ist nicht immer einfach.»

Nicht mehr zeitgemäss findet Conti die 26 kantonalen Gesundheitssysteme in den Kantonen. «Die Bevölkerung nimmt Kantons- und Landesgrenzen kaum noch wahr. Wir müssen künftig in Gesundheitsversorgungs-Regionen denken und planen.» Er ist für eine national koordinierte Gesundheitspolitik zwischen Bund und Kantonen. Weniger und vor allem spezialisierte Spitäler solle es geben. Fachpersonen bräuchten keine Angst vor Entlassungen zu ha- ben, denn sie würden dringend gebraucht werden. Investieren müsse die Politik auch in die Ausbildung: Junge Leute sollten zum Beispiel schon ab der Volksschule für den Pflegeberuf sensibilisiert und später nicht als billige Arbeitskräfte missbraucht werden.

Einig waren sich die Experten: Es braucht eine intelligente Regulierung, aber keiner konnte sagen, was intelligent bedeutet. «Die Bevölkerung will keine Einheitskasse, sie will wählen können, aber nicht zu viel dafür ausgeben», sagte Carlo Conti. «Ich weiss nicht, wie wir diese Quadratur des Kreises lösen können.» So viel Wettbewerb wie möglich und so wenig Regulierung wie nötig, forderte Ruth Humbel, CVP Nationalrätin und Mitglied der Kommission für soziale Sicherheit und Gesundheit des Nationalrats. Das «Modell Einheitskasse» führe in die Staatsmedizin, ein freiheitliches Gesundheitswesen erfordere dagegen Transparenz, klare Spielregeln und (Mit-)Verantwortung aller Akteure. Auch wie viel Wettbewerb gut ist, wurde kontrovers diskutiert. «Wenn im Kanton Luzern 700 Hüft-Operationen pro Jahr durchgeführt werden, müssten Angebote eingeholt werden, wer die am besten und günstigsten macht - das wäre wirklicher Wettbewerb», sagte Johann Steurer. «Aber das wird bisher nicht gemacht.» Die Sicht des Patienten sei dabei aber auch wichtig, sagte Carlo Conti. «Jeder Konsument im freien Markt wird sein Produkt nicht wechseln, wenn er damit zufrieden ist. Darum funktioniert das Modell mit den 700 Hüften nicht.»

Die grundsätzliche Frage bleibe, so Thomas F. Lüscher, was man im Gesundheitswesen erreichen wolle. «Wenn ich als Arzt einem Patienten unnütze Therapien anbiete, hat der trotzdem das Gefühl: So ein toller Doktor, der kümmert sich um mich. Dabei erhöht das nur die Kosten.» Er beobachte in der letzten Zeit eine Tendenz zur «Übertherapie», was sich auch in den immer neuen Diagnosen widerspiegelt, die manche Ärzte meinen behandeln zu müssen. «Irgendwann behandeln wir dann Alter als Diagnose», sagte Lüscher scherzhaft.

Konkrete Vorschläge für Ärzte, wie Innovationen im Gesundheitswesen effizienter umgesetzt werden können, präsentierte Adriaan Ruijs, Managing Director bei MSD Merck Sharp \& Dohme AG in Luzern. Neue Medikamente nützen beispielsweise nur, wenn Patienten sie auch einnehmen. Sind Patienten nicht adhärent, kann das die Ausgaben um ein Vielfaches steigern. Vielleicht würde die Adhärenz deutlich steigen, wenn Patienten mehr Verantwortung bekämen und eine aktivere Rolle für ihre Gesundheit und bei Entscheidungen übernehmen würden. Doch da sind auch die Ärzte gefragt, dass sie sich mehr Zeit nehmen für Erklärungen. Sorgen machten den Experten, dass viele junge Mediziner den klassischen Arztberuf aufgeben oder nicht als Niedergelassene arbeiten wollen. und wenn sie Arzt werden, dass sie dann nicht in die Praxis wollen. «Frage ich meine Assistenten, will kaum einer eine kardiologische Praxis», erzählte Lüscher. «Die meisten wollen Leitende oder Chefärzte werden.» Vielleicht findet der nächste Roundtable eine Lösung. 